\title{
Large scale direct galaxy collision simulations with central supermassive binary black holes
}

\author{
Margaryta Sobolenko ${ }^{1}$, Peter Berczik ${ }^{2,1,4}$ and Rainer Spurzem ${ }^{2,3,4}$ \\ ${ }^{1}$ Main Astronomical Observatory, National Academy of Sciences of Ukraine, 27 Akademika \\ Zabolotnoho St., 03680, Kyiv, Ukraine \\ email: sobolenko@mao.kiev.ua \\ ${ }^{2}$ National Astronomical Observatories of China, Chinese Academy of Sciences, 20A Datun \\ Rd., Chaoyang District, 100012, Beijing, China \\ ${ }^{3}$ Kavli Institute for Astronomy and Astrophysics, Peking University, Beijing 100871, China \\ ${ }^{4}$ Astronomisches Rechen-Institut, Zentrum für Astronomie, University of Heidelberg, \\ Mönchhofstrasse 12-14, 69120, Heidelberg, Germany
}

\begin{abstract}
We present a set of, large scale direct $N$-body simulations of the galaxy collision with the central Supermassive Black Hole Binary (SMBHB) system. Based on our simulations which include the accurate Post Newtonian (PN) relativistic dynamical corrections we can estimated the merging time for the real astrophysical object. Each galaxy initially was represented as a set of particles (up to $\mathrm{N}=500 \mathrm{k}$ ) with Plummer distribution. The SMBHBs system is described using the two special high mass, i.e. "relativistic", particles. The interaction between these two particles have an extra PN correction terms (up to 3.5PN). Merging time upper limit was obtained for the closely interacting galaxy system NGC 6240 .
\end{abstract}

Keywords. black hole physics, methods: $N$-body simulations, galaxies: nuclei, individual (NGC6240)

\section{Introduction}

After first discovering the AGN it was proposed that central power engine of this objects can be the massive black holes (Lynden-Bell 1969; Lynden-Bell \& Rees 1971; Wolfe \& Burbidge 1970; Longair 1996). Nowadays we know that at the cores of many active galaxies indeed we have a massive black holes (Ferrarese \& Ford 2005; Collin 2006). As an close example we can see our Galaxy, which contains the massive central black hole with the mass $M_{\bullet} \approx 4 \times 10^{6} \mathrm{M}_{\odot}$ (Gillessen et al. 2009). Usually the central supermassive black holes $(\mathrm{SMBH})$ have a mass range $10^{6}-10^{9} \mathrm{M}_{\odot}$.

Mass of the SMBH correlate with various physical parameters of the host galaxies stellar spheroid. The most clear correlations we can see are between the SMBH mass and the bulge luminosity $L_{\mathrm{bul}}$, the bulge mass $M_{\mathrm{bul}}$, the bulge velocity dispersion $\sigma$, the mass of the galaxy $M_{\mathrm{gal}}$, and even the Sérsic index of the surface brightness profile (Hartmann et al. 2014, reference therein).

During their evolution galaxies can merge with central BHs and can create also galaxies with SMBHB. At the first stage (Begelman et al. 1980) the separation between components can shrink due to dynamical friction in the stellar background and later can eventually form the "hard" binary. Further the binary can become more hard due to the three-body scattering of single stars, mainly inside the influence radius of the SMBHB system. At the last stage of merging the binary relativistic (with the emission of gravitational waves) in-spiral can lead to the final fast coalescence of the SMBHB.

We can observe the SMBHB at the different stages of merging as different objects: as 

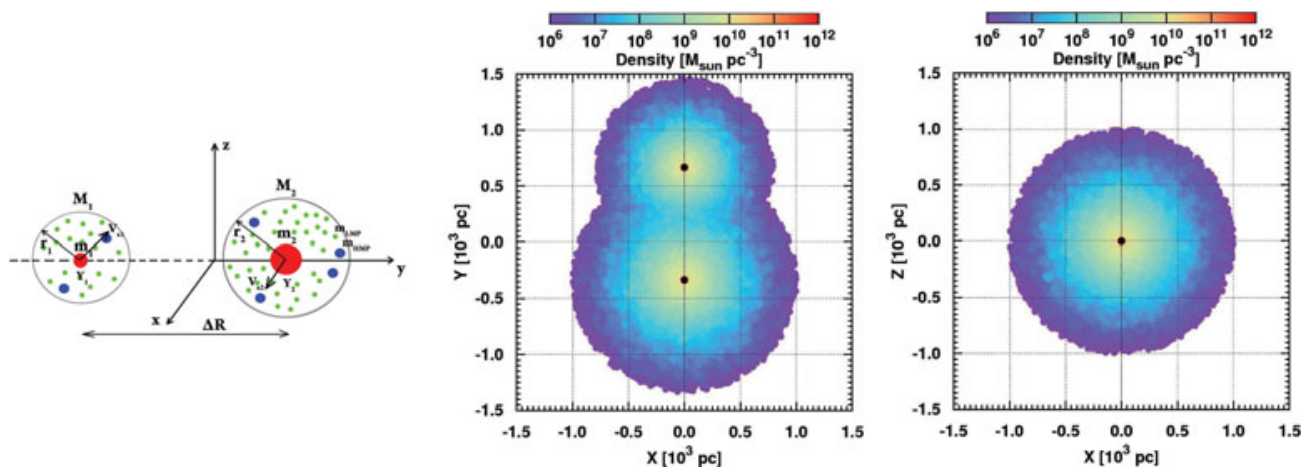

Figure 1. left panel: Initial configuration for system with two BHs at the centres. middle panel: Density cut at the XY plane at view. right panel: Density cut at the XZ plane at view.

SMBH at the galaxy pairs $(\Delta r \sim 10-100 \mathrm{kpc})$, as dual SMBHs $(\Delta r \sim 1 \mathrm{kpc})$, as binary SMBHs $(\Delta r<\mathrm{pc})$ or as a recoiling SMBHs after merging. This type of classification, of course, is quite arbitrary. For all the objects that we observe now and generally call as "binary SMBHs" with the separation more than $1 \mathrm{pc}$, we decide to use the term "SMBH binary (SMBHB)".

One of the first discovered SMBHB was a interacting galaxy NGC6240 (Komossa et al. 2003) situated at $\mathrm{z}=0.0243$ (Solomon et al. 1997). The two sources at the LINER (Nakai et al. 2002; Véron-Cetty \& Véron 2010, see references) galaxy are resolved in hard Xrays by the Chandra X-Ray Observatory with separation $\Delta \theta=1.56^{\prime \prime}$ (corresponding $\Delta r=0.756 \mathrm{kpc}$, assumed $H_{0}=68 \mathrm{kms}^{-1} \mathrm{Mpc}^{-1}, 1^{\prime \prime}=504 \mathrm{pc}$ ). The lower limit for south-western BH mass $M_{\bullet \mathrm{S}}=8.7 \pm 0.3 \times 10^{8} \mathrm{M}_{\odot}$ was measured using stellar kinematics (Medling et al. 2011; Engel et al. 2010). The dynamical mass of the nuclei should be $M_{\mathrm{dyn}}=2-8 \times 10^{9}$ from the stellar velocity field in central $500 \mathrm{pc}$ (Tecza et al. 2000).

\section{Numerical model and initial conditions}

For simulations we took the next physical value for system: total mass of the galaxies is $M_{\text {tot }}=1.3 \times 10^{11} \mathrm{M}_{\odot}$, total masses of the SMBHB are $M_{\bullet \text { tot }}=1.3 \times 10^{9} \mathrm{M}_{\odot}=1 \% M_{\mathrm{tot}}$ and $M_{\bullet \text { tot }}=2.6 \times 10^{9} \mathrm{M}_{\odot}=2 \% M_{\text {tot }}$, BHs and galaxies mass ratio is $q=M_{\bullet \mathrm{sW}} / M_{\bullet \mathrm{NE}}=$ 0.5 , initial separation is $\Delta R=1 \mathrm{kpc}$, initial eccentricity is $e_{0}=0.5$. We represent two galaxies as two Plummer (non-rotating) spheres with two special particles at the centres called BHs (Fig. 1). Also spheres contain another two type of particles called "light mass particles" (LMP) with $90 \% N_{\text {tot }}$ and "high mass particles" (HMP) with $10 \% N_{\text {tot }}$. The mass ratio between HMP and LMP was 1:9. We set the total number of the particles as $N_{\text {tot }}=500 \mathrm{k}$.

We use the publicly available $\varphi$ GPU code $\dagger$ (Berczik et al. 2011, 2013) with $N$ body $4^{\text {th }}$ order Hermite integration of the motions for all the particles using the blocked hierarchical individual time step scheme. This Hermite scheme requires us to know the acceleration and its first time-derivative, called jerk, including the $\mathcal{P} \mathcal{N}$ corrections. In our $\varphi$ GPU code we use the generalized "Aarseth" type criteria for the timepstep definition (Nitadori \& Makino 2008):

$$
\Delta t=\eta_{p}\left(\frac{A^{(1)}}{A^{(p-2)}}\right)^{1 /(p-3)},
$$

$\dagger$ ftp://ftp.mao.kiev.ua/pub/berczik/phi-GPU/ 

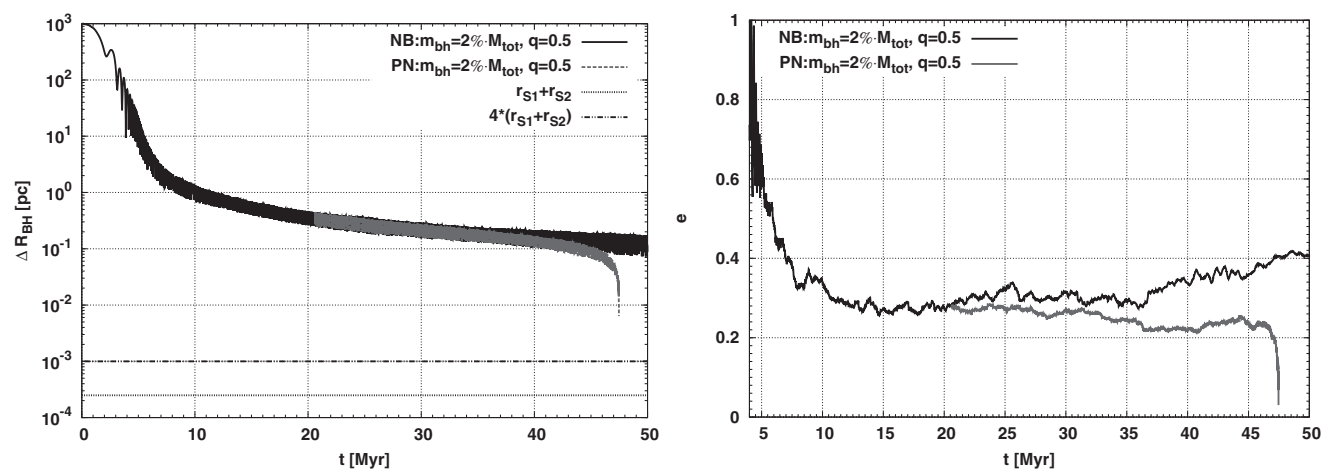

Figure 2. Evolution of separation between the SMBHs (left panel) and eccentricity (right panel) for model with $M_{\bullet \text { tot }}=2 \% M_{\text {tot }}$

where

$$
A^{(k)}=\sqrt{\left|\boldsymbol{a}^{(k-1)}\right|\left|\boldsymbol{a}^{(k+1)}\right|+\left|\boldsymbol{a}^{(k)}\right|^{2}} .
$$

Here, $\boldsymbol{a}^{(k)}$ is the $k^{\text {th }}$ derivative of acceleration, $p$ is the order of the integrator, $\eta_{p}$ is the accuracy parameter. For $4^{\text {th }}$-order Hermite scheme the timestep looks like:

$$
\Delta t=\eta_{4} \frac{A^{(1)}}{A^{(2)}}
$$

where

$$
A^{(1)}=\sqrt{|\boldsymbol{a}|\left|\boldsymbol{a}^{(2)}\right|+\left|\boldsymbol{a}^{(1)}\right|^{2}}, \quad A^{(2)}=\sqrt{\left|\boldsymbol{a}^{(1)}\right|\left|\boldsymbol{a}^{(3)}\right|+\left|\boldsymbol{a}^{(2)}\right|^{2}} .
$$

For all our runs we use the $\eta_{4}=0.1$ parameter for our two black holes. The gravitational softening $\varepsilon$ used in form:

$$
\varepsilon_{i j}^{2}=\alpha\left(\varepsilon_{i}^{2}+\varepsilon_{j}^{2}\right) / 2
$$

where $\varepsilon_{\bullet}=0, \varepsilon_{\mathrm{HMP}}=10^{-4}, \varepsilon_{\mathrm{LMP}}=10^{-5}$. If one of the interacting particle was a $\mathrm{BH}$ we use the coefficient $\alpha=10^{-4}$, for other cases $\alpha=1$.

\section{Results and discussion}

We use two distinct hardening regimes for obtaining the merging time for object NGC6240. First classical regimes caused by hardening from stellar-dynamical effects $\left(S_{\mathrm{NB}}\right)$ (Khan et al. 2012). The next regime is a relativistic regime with hardening by gravitational waves emission $\left(S_{\mathrm{GW}}\right)$. At the classical regime we neglect the $S_{\mathrm{GW}}$ ) and estimate $S_{\mathrm{NB}}=$ const. GW emission start dominate at time when $S_{\mathrm{NB}}=S_{\mathrm{GW}}$. We start to calculate the $S_{\mathrm{GW}}$ at time when $S_{\mathrm{GW}}=0.03 \% S_{\mathrm{NB}}$. At Figure 2 we show the relative separation $\Delta R$ between the two black holes during a galaxy merger and eccentricity $e$ evolution for model with $M_{\bullet \text { tot }}=2 \% M_{\text {tot }}$. Eccentricity stays almost constant during a whole period of time.

The estimated merging time for model with $M_{\bullet \text { tot }}=1 \% M_{\mathrm{tot}}$ is $T_{\mathrm{merge}} \%=40 \mathrm{Myr}$ and for the model with $M_{\bullet \text { tot }}=2 \% M_{\text {tot }}$ is $T_{\text {merge } 2 \%}=48$ Myr (Fig. 3).

\section{Acknowledgements}

MS \& PB thanks the International Astronomical Union for the grant support which gave the opportunity to him participate on the IAU Symposium No. 312 in Beijing-2014. 


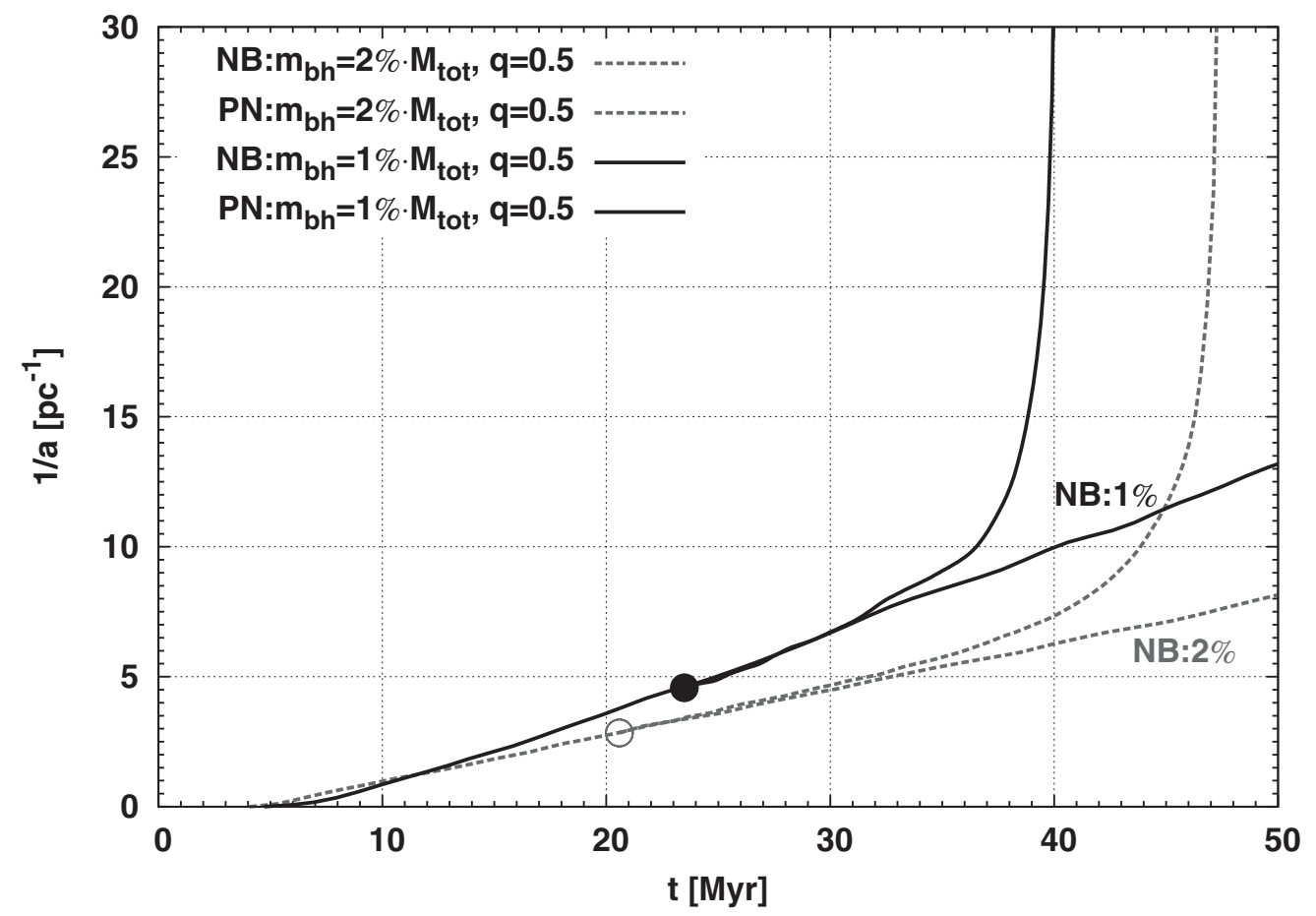

Figure 3. Evolution of SMBH binary inverse semi-major axis for two models with

$$
M_{\bullet \text { tot }}=1 \% M_{\text {tot }} \text { and } M_{\bullet \text { tot }}=2 \% M_{\text {tot }}
$$

\section{References}

Begelman, M. C., Blandford, R. D., \& Rees, M. J. 1980, Nature, 287, 307

Berczik, P., Spurzem, R., Wang, L., Zhong, S., \& Huang, S. 2013, in Third International Conference "High Performance Computing", HPC-UA 2013, p. 52-59, 52-59

Berczik, P., Nitadori, K., Zhong, S., et al. 2011, in International conference on High Performance Computing, Kyiv, Ukraine, October 8-10, 2011., p. 8-18, 8-18

Collin, S. 2006, in American Institute of Physics Conference Series, Vol. 861, Albert Einstein Century International Conference, ed. J.-M. Alimi \& A. Füzfa, 587-595

Engel, H., Davies, R. I., Genzel, R., et al. 2010, A\&A A, 524, A56

Ferrarese, L., \& Ford, H. 2005, Space Sci. Rev., 116, 523

Gillessen, S., Eisenhauer, F., Trippe, S., et al. 2009, Astrophys. J., 692, 1075

Hartmann, M., Debattista, V. P., Cole, D. R., et al. 2014, Mon. Not. R. Astron. Soc., 441, 1243

Khan, F. M., Preto, M., Berczik, P., et al. 2012, Astrophys. J., 749, 147

Komossa, S., Burwitz, V., Hasinger, G., et al. 2003, Astrophys. J., Lett., 582, L15

Longair, M. S. 1996, Our evolving universe (Cambridge, New York: Cambridge University Press) Lynden-Bell, D. 1969, Nature, 223, 690

Lynden-Bell, D., \& Rees, M. J. 1971, Mon. Not. R. Astron. Soc., 152, 461

Medling, A. M., Ammons, S. M., Max, C. E., et al. 2011, Astrophys. J., 743, 32

Nakai, N., Sato, N., \& Yamauchi, A. 2002, Publ. Aston. Soc. Jpn., 54, L27

Nitadori, K., \& Makino, J. 2008, N. Astr., 13, 498

Solomon, P. M., Downes, D., Radford, S. J. E., \& Barrett, J. W. 1997, Astrophys. J., 478, 144

Tecza, M., Genzel, R., Tacconi, L. J., et al. 2000, Astrophys. J., 537, 178

Véron-Cetty, M.-P., \& Véron, P. 2010, A\& A, 518, A10

Wolfe, A. M., \& Burbidge, G. R. 1970, Astrophys. J., 161, 419 